\title{
Concerning the Hallmark of a Discrimination
}

\author{
William C. Stebbins and David B. Moody \\ Psychology Department and the Kresge Hearing Research Institute \\ University of Michigan
}

Principles of Psychology (Keller \& Schoenfeld, 1950) was articulate, rigorous, systematic, and a most effective teacher. But it was more than that. It was a book full of good research ideas. The title of this article comes from our favorite chapter on discrimination ("extinction is the hallmark of-") (Keller \& Schoenfeld, 1950, p. 119). Book and chapter influenced us tremendously and led us on our careers, first in animal psychophysics, and then into some more arcane problems in what we call animal perception (Stebbins, in press; Stebbins $\&$ Berkley, in press). More about that later. We dedicate this paper to Fred Keller and Nat Schoenfeld because theirs was such a strong determining influence, and because, between them, they created a superb atmosphere for research and scholarship at Columbia in the 1950s and $60 \mathrm{~s}$. We would be remiss if we didn't mention Fred Skinner's hand in all of this. Fred Keller and Nat Schoenfeld's intense and scholarly graduate seminars made ample use of Behavior of Organisms (Skinner, 1938) and many of Fred Skinner's papers.

Our objective here is to present an encapsulated review of our research because what we have done over some 30 plus years is a direct outgrowth of Principles, at least as we read it. The story starts with discriminative reaction time: "By gradually eliminating the longer latencies through extinction while continuing to reinforce the shorter ones, we ultimately reach a latency that is minimalbeyond which selective reinforcement is no longer effective" (Keller \& Schoenfeld, 1950, p. 145). Minimal reaction time could be shown to vary with such parameters as schedule and amount of reinforcement. But more interestingly it could be utilized as a way of overcoming the language barrier between human and animal subject, thus permitting other ani- mals to make perceptual judgments about psychological attributes of stimuli such as loudness and brightness. We made the assumption that equal reaction times represented equal measures of sensory effect. Thus, for example, equal latencies of response to different frequencies of acoustic stimulation could be considered judgments of equal loudness. Using reaction time allowed us to circumvent the difficult reinforcement question. While one could set up, a priori, reinforcement contingencies for equal physical intensities or levels of stimulation, there is no way that this can be done for such psychological attributes as loudness and brightness. To attempt to do so would prejudge the animal's perceptual continuum and build in an experimenter-devised sensory scale. In fact this may have happened in Herrnstein and Van Sommers' (1962) otherwise quite elegant experiment on brightness scaling in pigeons and the power law published in Science.

We took the basic reaction time procedure into threshold psychophysics with animals; lever depression was the observing response and lever release the reporting response: "Since the capacities of infra-human organisms cannot be gauged by way of verbal responses, we resort to the basic procedure of forming a discrimination" (Keller and Schoenfeld, 1950, p. 134). We did just that and will spare you the fine details. Our purpose was to acquire a better understanding of the acoustic sense of animals: "the investigation of the discriminative capacities of organisms" (Keller \& Schoenfeld, 1950, p.130). Our purpose was twofold and made contact with two other disciplines-evolutionary biology and neurophysiology. First, we sought to better understand the evolution of hearing and the auditory system by examining hearing in a variety of animals near the line of descent to man. Second, we offered the physiologists and 


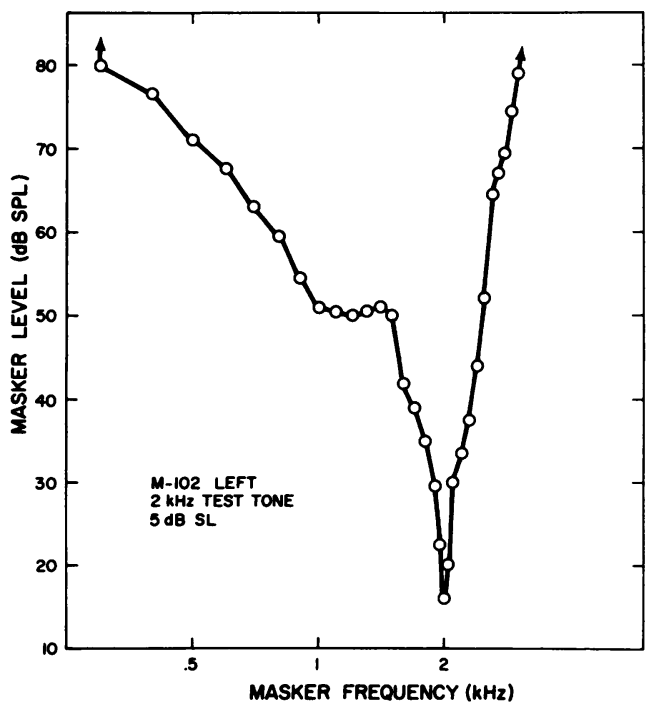

Figure 1. Psychophysical tuning curve for a macaque monkey. The test tone remained at $2 \mathrm{kHz}, 5$ $\mathrm{dB}$ above threshold, while the masking tone was presented at the frequencies indicated. The level of the masking tone at each frequency at which the test tone could be detected $50 \%$ of the time is a measure of the frequency selectivity of the auditory system. From "Comparative Behavioral Toxicology" by W. C. Stebbins and D. B. Moody, 1979, Neurobehavioral Toxicology and Teratology, 1 (Suppl. 1), pp. 33-44. Copyright 1979 by Pergamon Press. Reprinted by permission.

anatomists an opportunity to better interpret the "what for" or the function of all those mechanisms they continued to discover in the ear and central nervous system. More about this second purpose now; more about the first one later. With the power of operant conditioning we could offer these other biological scientists the precision and reliability to which they were accustomed in their own discipline with microscope and microelectrode.

There are drugs (certain antibiotics) that selectively destroy inner ear receptors in a very predictable and progressive manner over time and thus permit a before, during, and after drug measure of auditory acuity. The impairment is very similar to age-related hearing loss, with the loss occurring first at the higher frequencies and subsequently at the midrange and finally at the low frequencies. A final, preterminal measure of hearing can therefore be related to the pattern and distribution of missing receptor cells in the cochlea of the inner ear as determined postmortem by microscopy and can perhaps reveal something about the function of those receptor cells in the normal ear. To complicate matters further, there are in the inner ear, as in the eye, two kinds of receptor cells that can be distinguished both anatomically and physiologically. One drug (given to the macaque monkey) destroys both cell types; a second drug (given to patas monkey or guinea pig) destroys one cell type while substantially sparing the other. By measuring auditory thresholds in these impaired subjects with our positive reinforcement procedure at many pure-tone frequencies, we are able to provide a meaningful statement about the nature of frequency coding in the mammalian inner ear and thus offer a functional use for those anatomical and physiological mechanisms.

We took the whole matter a step further by asking a still more intricate question. Given the behaviorally meaningful acoustic frequency-to-place transformation in the inner ear, what are the width and other relevant characteristics of the frequency filter along the basilar membrane of the inner ear? The answer called for a somewhat more elaborate psychophysical procedure that required a masking paradigm with one pure tone on top of another, but the behavioral procedure was effectively the same with the exception that the animal was discriminating one sound from another rather than sound from silence. In the course of the experiment one pure tone (the test tone) remained at a set frequency and sound level (for example, $4,000 \mathrm{~Hz}$ at $10 \mathrm{~dB}$ above threshold). The masking tone was then varied over the whole audible region at numerous values of frequency and sound level, and the correct detection of the test tone in the presence of the masker was reinforced. Simply stated, the threshold was that level of the masker at which the subject could detect the test tone half of the time (see Figure 1). The resultant function, which appears graphically as a downward-pointing wedge, is known as a psychophysical tuning curve and is a measure of the frequency selectivity of 


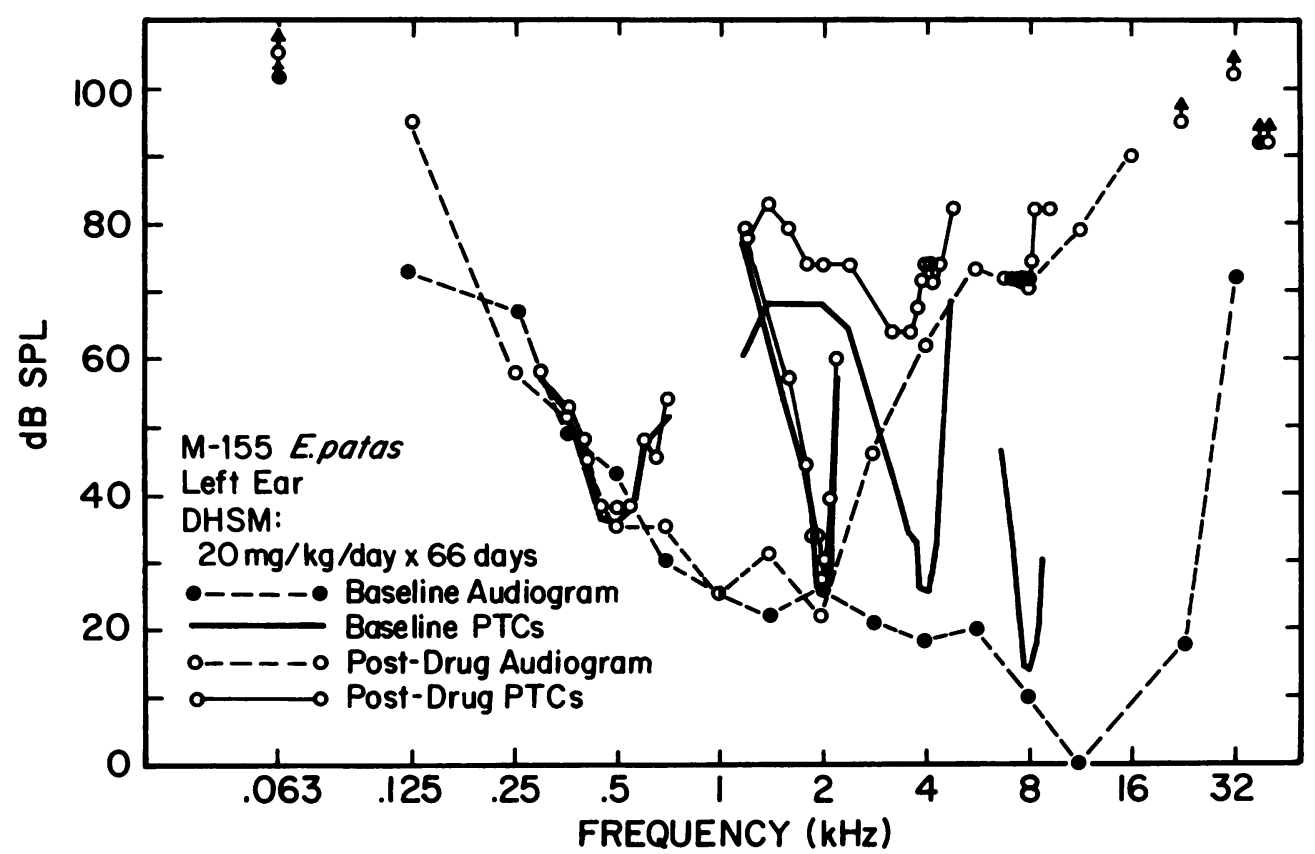

Figure 2. Direct comparison of baseline (pre-drug) and post-drug pure-tone threshold curves (audiograms) and psychophysical tuning curves for a patas monkey. The impairment in auditory sensitivity is shown in the post-drug audiogram that rises sharply above $2 \mathrm{kHz}$ and in the psychophysical tuning curves that are elevated and broadened for test tone frequencies of 4 and $8 \mathrm{kHz}$ while those at $500 \mathrm{~Hz}$ and 2 $\mathrm{kHz}$ remain normal. From "Effects of Outer Hair Cell Loss on the Frequency Selectivity of the Patas Monkey Auditory System" by D. W. Smith, D. B. Moody, W. C. Stebbins, and M. A. Norat, 1987, Hearing Research, 29, pp. 125-138. Copyright 1987 by Elsevier Science Publishers BV. Reprinted by permission.

the auditory system. Its resemblance to the physiological tuning curve for a single auditory neuron of the eighth nerve is striking and leads to the conclusion that this form of selectivity, at least, is handled in the auditory periphery. If once again we use drugs that damage the peripheral auditory system, we immediately see the loss of frequency selectivity in the change in shape of the tuning curve at those frequencies at which hearing is impaired in the auditory threshold function. The complicated function in Figure 2 (Smith, Moody, Stebbins, \& Norat, 1987) is simply the auditory threshold function and the psychophysical tuning curve for one animal before and after drug treatment. It is no more than an elaborate variation on the hallmark theme-extinction of $S^{\Delta}$ responding. The experimental question is a disarmingly elementary one; the paradigm has evolved directly from its simplest but most general form as expressed in Principles of
Psychology. But the answer is very revealing regarding the functional properties of the nervous system. The behavioral approach and methodology are essential if we are to understand, properly, nervous system function in comparative neurobiology.

In these psychophysical experiments on what Keller and Schoenfeld have suitably named "the discriminative capacities of organisms," we had pushed our animal subjects to their limits, and they had proven to be immensely reliable observers or listeners. But, on an everyday basis, animals are not operating out at the margin of their sensory resolving power. They are not routinely forced to their limits. What an animal can do under marginal or extreme conditions is not always the same as what it does do under normal or usual circumstances. For example, from Keller and Schoenfeld (1950, p. 155), "generalization within classes and discrimination between classes-this is 
the essence of concepts." We and other animals categorize, and it is usually efficacious to do so. Whether we generalize or discriminate in a particular instance will often depend on the context. In other words, while we might normally generalize, if pushed to the edge, we can discriminate. As an illustration in the perception of speech, we generalize across very different acoustic waveforms as in "you all" versus "yawl" but have little difficulty discriminating between them if the situation requires it.

The distinction between "can do" and "does do" is, we believe, a useful one. There are some interesting and challenging problems for the experimental analysis of behavior in determining what an animal does do under ordinary circumstances when the stimuli to which it attends are not at threshold but in a broad dynamic range above it. If we now eschew pure tones in favor of those complex signals that are part of an animal's acoustic communication system, we have increased the challenge, but we have also improved the "ecological validity" of the preparation. By ecological validity we mean no more or no less than the use of the natural species signals that animals employ in communicating with conspecifics. The major question is still a perceptual one and we have referred to it in the context of evolutionary biology earlier in the paper and as a major goal of our research. It is, we suggest, what Keller and Schoenfeld referred to (and with their usual caution it came in quotation marks) as "higher units in perception" (Keller \& Schoenfeld, 1950, p. 153): "In the laboratory, we seek to isolate the $S^{D}$ s wich enter into complex discriminations, although we know that in ordinary experience they are not so isolated. But we would also like to know how they become integrated."

More about the rationale for this approach and some of the disciplinary threads to which it is tied. Certainly one of the key questions driving this research concerns the evolution of communication and human speech and their relation to the evolution of the auditory system and hence to the perception of biological signals. The motor theory of speech perception suggests that "speech is special" (Liberman, 1982) and that there is a qualitative difference between humans and other animals in the perception of speech. Behavioral research with animals counters this suggestion by demonstrating that animals are capable of discriminating human speech sounds and in a categorical manner as do humans (Kuhl, 1986). But we are interested in how animals perceive their own communication sounds and what commonalities might exist between their perception of their own sounds and our perception of speech.

Results of field studies of acoustic communication in nonhuman primates by Peter Marler and his colleagues (Green, 1975) provided us with an extensive sampling of tape-recorded signals whose communicative and social function had been determined in field studies under natural conditions. Our question concerned the perception of these acoustic signals when both animal and signal had been removed to the laboratory away from their rich and elaborate natural environment. Could these animals then discriminate these signals when all other contextual cues had been removed? Would we be able to see any similarities to human speech perception, for example, in perceptual constancy or neural lateralization of species calls in nonhuman subjects? Finally, could we find important features in these complex stimuli that were responsible for the perceptual response and that might have conveyed some selective advantage in the course of evolution? Once more from Keller and Schoenfeld, "It is easy to show that when $\mathrm{S}^{\mathrm{D}}$ compounds are the basis of a response it is possible to obtain that response to a portion of the compound." And "redintegration is a case of generalization through partial identity" (Keller \& Schoenfeld, 1950, p. 152).

The Japanese monkey (Macaca fuscata) commonly utters a somewhat tonal call-the "coo" call-that rises and falls in frequency. The social function of the call varies depending on whether the frequency inflection occurs at the beginning or at the end of the call. An early inflec- 
tion (in the first two thirds of the call) most often denotes an animal out of visual range maintaining contact with the rest of the troop, and the call is answered by other troop members. An inflection late in the call (in the final third) usually signifies an estrus female soliciting a mate (see Green, 1975). The time of the frequency inflection is on a continuum and is thought to be analogous to the continua for phonemes in human speech perception such as "ba" and "da" which differ in the direction of a fast frequency sweep at the beginning of the signal. Our first experiment simply attempted to determine if the Japanese monkeys could discriminate between these two call types in the laboratory (Beecher, Peterson, Zoloth, Moody, \& Stebbins, 1979). For stimuli we used the actual signals from the field tapes. The animals picked up the discrimination very quickly and were soon discriminating between many exemplars of the two call types in spite of considerable differences in the acoustic signal occasioned by such things as different callers, distance of the caller from the microphone, and so on. However acquired, there seems to be a kind of perceptual constancy for these calls. In the same discrimination paradigm we presented the calls to animals from a different species, same genera, and found that they had considerable difficulty acquiring the discrimination. This suggests that in some way, yet unknown, these calls were species typical.

In the course of these experiments we had the monkeys wear earphones so that we could deliver the signals to right or left ear independently. Our objective was to check for discriminative accuracy by ear and thus by cerebral hemisphere. Our results confirmed a right ear and, by implication, a left hemisphere superiority for the call discrimination in all of the Japanese monkeys and only in one of the controls (Peterson, Beecher, Zoloth, Moody, \& Stebbins, 1978). We felt more confident when these results were confirmed and extended by the Heffners, who showed further that a severe although transient discriminative decrement for these calls was effected following lesions of the left temporal lobe but not of the right (Heffner \& Heffner, 1984). We had thus uncovered two characteristics of the perception of monkey calls that are shared by human speech perception-perceptual constancy and left hemisphere dominance.

A third common feature of humans' perception of speech is categorization, or what we used to call concept formation. Of course both humans and other animals (even birds) categorize phonemes such as "ba" and "da," suggesting that this property of speech perception is not uniquely human and may reflect a more general property of animal auditory or central nervous systems. It was our intention to pursue this question using an example from the Japanese monkeys' own communicative repertoire to find out whether other animals categorize or conceptualize within their own system of communication. "Ba" and "da" reside at the ends of a continuum represented by a fast frequency change at the beginning of the signal, at one end a rise in frequency ("ba") and at the other a fall ("da") with continuous gradation between. Subjects generalize within each half of the continuum but discriminate between the two halves; in more colloquial language they identify everything in one half as "ba" and in the other half as "da." We put the question to the Japanese monkeys using the coo calls varying along a continuum with the early frequency inflection (smooth early high, SEH) at one end and the late inflection (smooth late high, SLH) at the other (May, Moody, \& Stebbins, 1989). The question was "Do you categorize the coo call continuum similarly to the way in which the "ba"-"da" continuum is categorized?" Or, in other words, "Do you generalize within an early or within a late coo call class but discriminate between members of the two classes?"

Asking such a question of other animals raises some interesting methodological issues. It is the "does do" as opposed to the "can do" question that we were asking. On the basis of psychophysical data we were quite sure that the animals could discriminate between the 


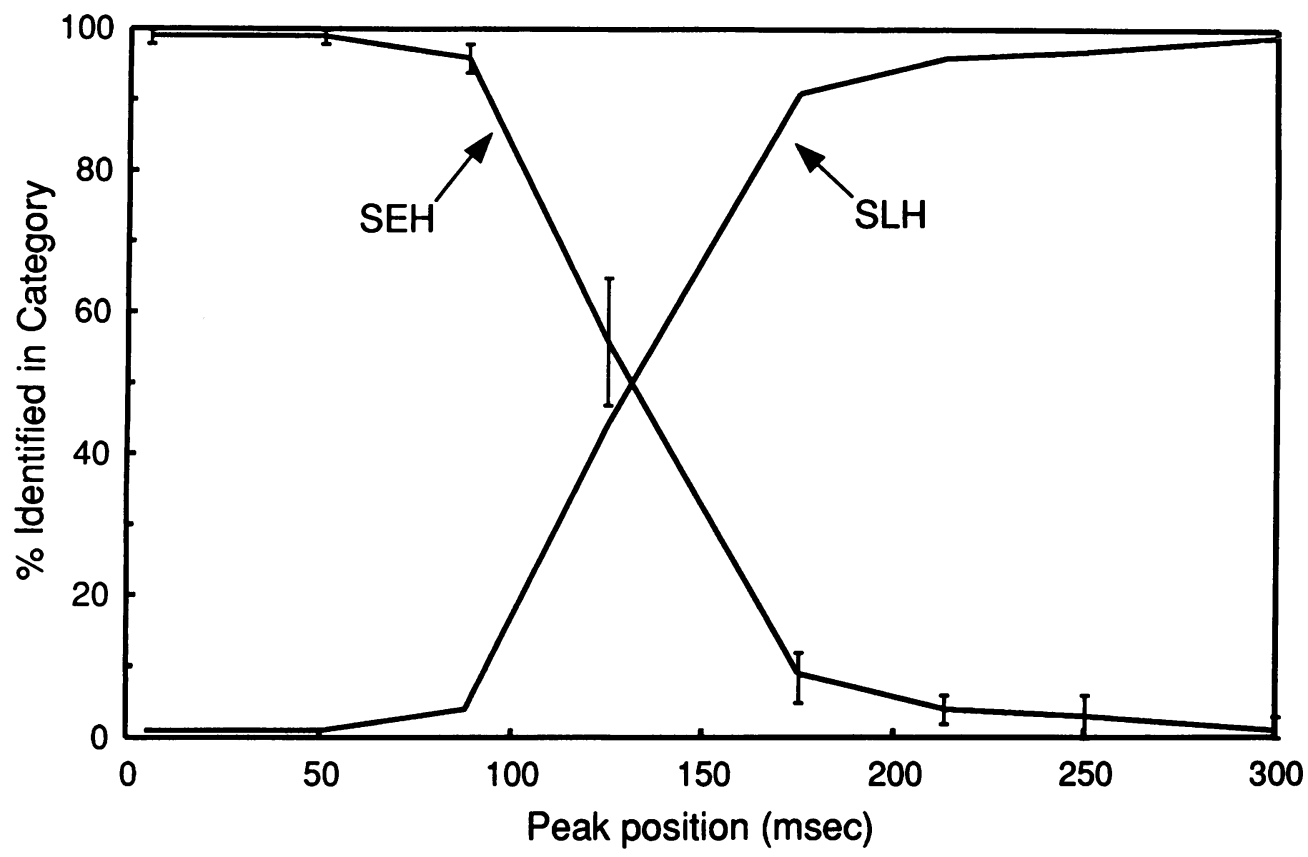

Figure 3. Identification of synthetic "coo" calls. Data are presented as the mean and the standard error of the mean for four subjects (Japanese monkeys). A category boundary, as indicated by the sharp transition in the behavioral response, was observed near the midpoint of the function. Although most of the synthetic stimuli were placed in only one of the two vocal categories (smooth early high or smooth late high), responses to the stimulus with a $125-\mathrm{ms}$ peak position were more equivocal. From "Categorical Perception of Conspecific Communication Sounds by Japanese Macaques, Macaca fuscata" by B. J. May, D. B. Moody, and W. C. Stebbins, 1989, Journal of the Acoustical Society of America, 85, pp. 837-847. Copyright 1989 by American Institute of Physics. Reprinted by permission.

signals within the two classes. We were interested in their treatment of the stimuli without explicit discrimination training. Would they discriminate or generalize within each class? Very briefly, we set up the previous discrimination between the two ends of the continuum. Responses to $\mathrm{S}^{\mathrm{D}}$ were reinforced $85 \%$ of the time, while responses to $\mathrm{S}^{\Delta}$ were followed by a brief time out.

We then very carefully computer synthesized a series of stimuli that covered the entire continuum from an early frequency inflection to one occurring near the end of the signal. On a very lean schedule we probed with the synthetic stimuli on the continuum, neither reinforcing responses to them nor following those responses with a time out. The original discrimination was maintained with the many examples of the taped natural calls. Casually stated, we wanted the animals' judgment on these intermediate stimuli without the experimenter's intervention. It was clear from the beginning that the synthetic stimuli near the ends of the continuum were perceived as natural calls by the monkeys. This was reassuring to the human experimenters who perceived them similarly. But the intriguing result, under the conditions of this experiment, was that the animals did categorize the stimuli as shown in Figure 3 , and in a very similar fashion to the manner in which speech sounds are categorized. They generalized to those stimuli with the frequency inflection early in the call as one class and to those stimuli with a late inflection as another class and discriminated between exemplars of the two classes.

Finally we considered the matter of redintegration (May, Moody, \& Stebbins, 1988). Are there features of these Japanese monkey coo calls that are particularly salient with regard to their percep- 

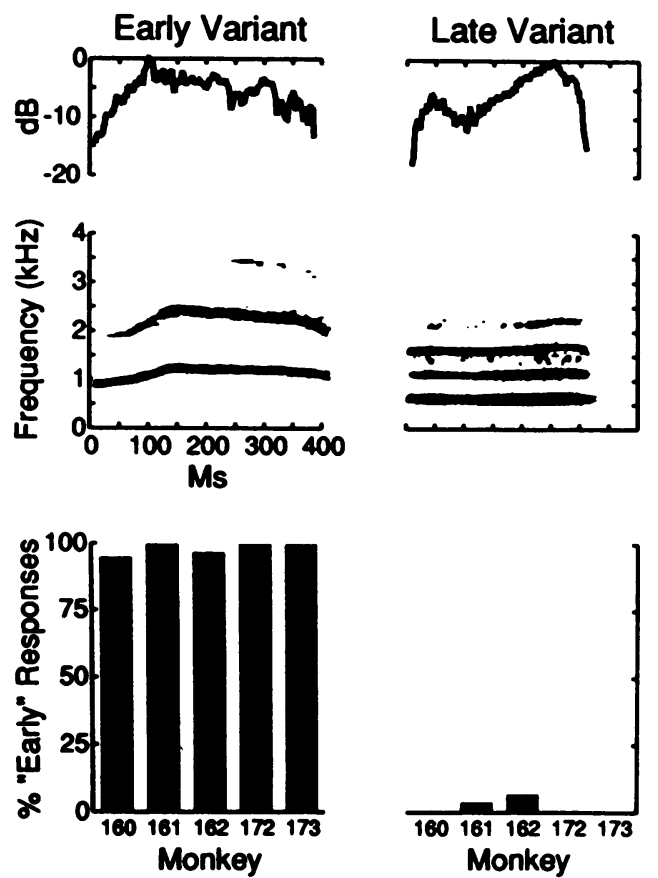

Figure 4. Sonogram for natural early (upper left) and late (upper right) inflected coo calls. The contours at the top of the panel represent the amplitude changes while those just below represent the frequency changes for the fundamental and harmonics. Percent "early" responses to the early inflected call (lower left) and percent "early" responses to the late inflected call (lower right). All animals responded to the early variant as an early inflected call but not similarly to the late variant. From "The Significant Features of Japanese Macaque Coo Sounds: A Psychological Study" by B. J. May, D. B. Moody, and W. C. Stebbins, 1988, Animal Behavior, 36, pp. 1432-1444. Copyright 1988 by Bailliere Tindall. Reprinted by permission.

tion? Our hypothesis based on the field data was that the frequency shift or inflection was important, but, since there were other variables that were also changing, we had to rule them out as critical. The only way to answer this question was to resort again to synthetic stimuli so that we could alter or degrade them easily, and, once again, we would use the generalization probe procedure that we had employed in the categorical perception experiment just described. Examples of natural stimuli shown in Figure 4 illustrate the basic paradigm and findings. In the upper panel of Figure 4 the amplitude contour of the signals lies over the frequency sonogram which indicates
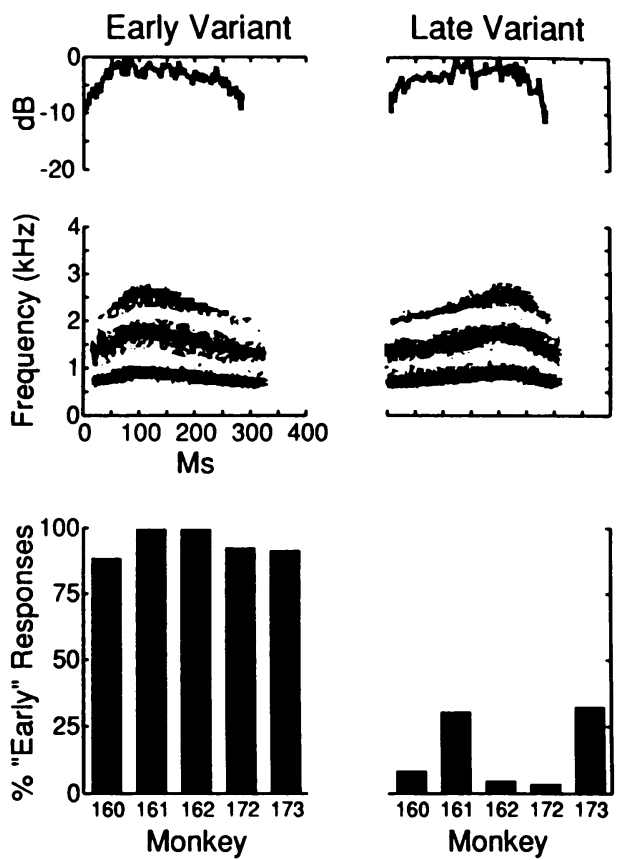

Figure 5. Sonogram for synthetic early (upper left) and late (upper right) inflected coo calls. The contours at the top of the panel represent the amplitude changes while those just below represent the frequency changes for the fundamental and harmonics. Responses to the calls in lower panels as described for Figure 4. Animals responded to the early variant as an early inflected call, but not similarly to the late variant. From "The Significant Features of Japanese Macaque Coo Sounds: A Psychological Study" by B. J. May, D. B. Moody, and W. C. Stebbins, 1988, Animal Behavior, 36, pp. 14321444. Copyright 1988 by Bailliere Tindall. Reprinted by permission.

the frequency changes with time. These were two new natural stimuli, one with an early frequency inflection, the other with a late inflection, that we first introduced as probes; the animals' response to them is seen in the histogram in the lower panel of the figure. They were readily accepted as just what they are. The call with the early frequency peak was responded to as an early inflected call, while the call with the late peak was almost never identified as an early inflected call. In Figure 5 is our computerized rendition of two prototypical coo calls (early and late inflected) synthesized in the laboratory. They appear to be perceived as natural calls.

Next are two modified synthetic calls 

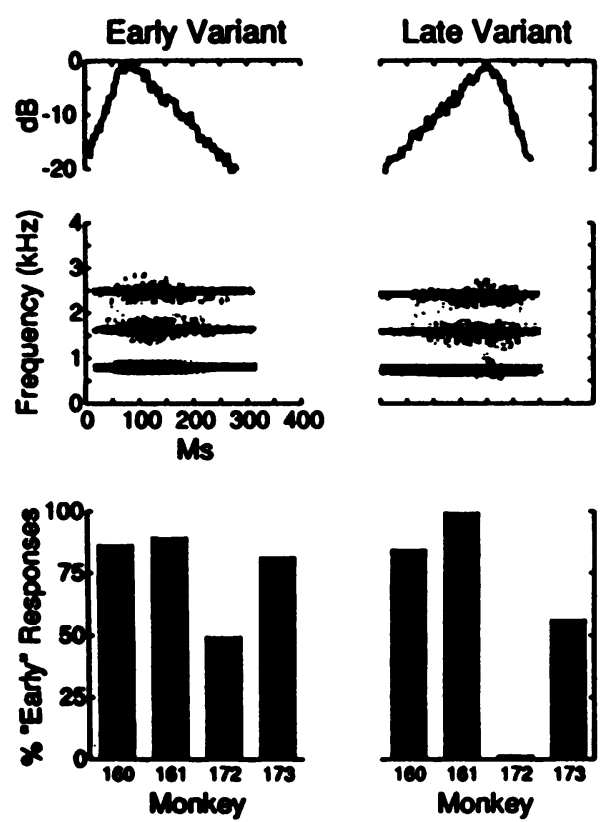

Figure 6. Sonogram for synthetic early (upper left) and late (upper right) inflected coo calls. The contours at the top of the panel represent the amplitude changes while those just below represent the frequency changes for the fundamental and harmonics. The frequency inflection has been eliminated although the amplitude change has been retained. Responses to the calls in lower panels as described for Figure 4. For the most part the animals responded similarly to both early and late variants. From "The Significant Features of Japanese Macaque Coo Sounds: A Psychological Study" by B. J. May, D. B. Moody, and W. C. Stebbins, 1988, Animal Behavior, 36, pp. 1432-1444. Copyright 1988 by Bailliere Tindall. Reprinted by permission.

where we have removed the frequency inflections but maintained the amplitude changes (see Figure 6). With one partial exception the animals do not discriminate between them. We then shortened the inflection at the beginning and end of the call and found to our surprise that our subjects discriminated poorly if at all between the two signals (see Figure 7). Finally we removed the frequency inflection entirely and what remained was a gradual glide either increasing or decreasing in frequency. As seen in Figure 8, the animals identified the decreasing glide almost all of the time as an early variant of the coo call and the increasing glide as a late variant as shown in the figure. Guardedly, then, since these are new data,
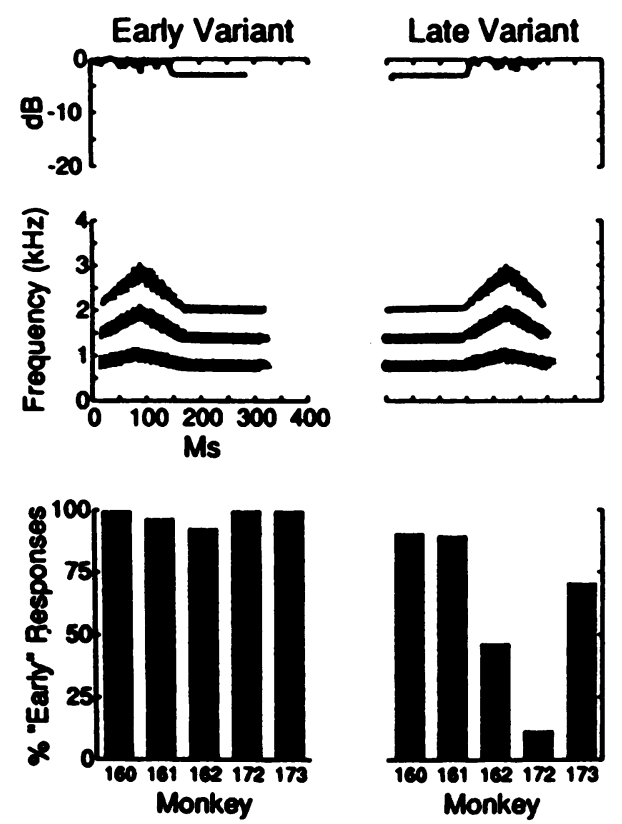

Figure 7. Sonogram for synthetic early (upper left) and late (upper right) inflected coo calls. The contours at the top of the panel represent the amplitude changes while those just below represent the frequency changes for the fundamental and harmonics. The frequency inflection has been shortened. Responses to the calls in lower panels as described for Figure 4. For the most part the animals responded similarly to both early and late variants. From "The Significant Features of Japanese Macaque Coo Sounds: A Psychological Study" by B. J. May, D. B. Moody, and W. C. Stebbins, 1988, Animal Behavior, 36, pp. 1432-1444. Copyright 1988 by Bailliere Tindall. Reprinted by permission.

and there are some holes that need plugging, we concluded that the frequency shift was an important information-bearing element in the call. This fits nicely with other evidence on the importance of this particular element (frequency shift) in vocalizations ranging from human speech to the echoranging signals of bats and there are some plausible reasons for its adaptiveness.

We have touched on some of the research that we have carried out in the past many years. Time and space constraints prevent us from delving more deeply into the procedures and some of the essential controls. There is no question but that the research was made possible by the rigorous kind of training that we had at Columbia from Fred Keller 

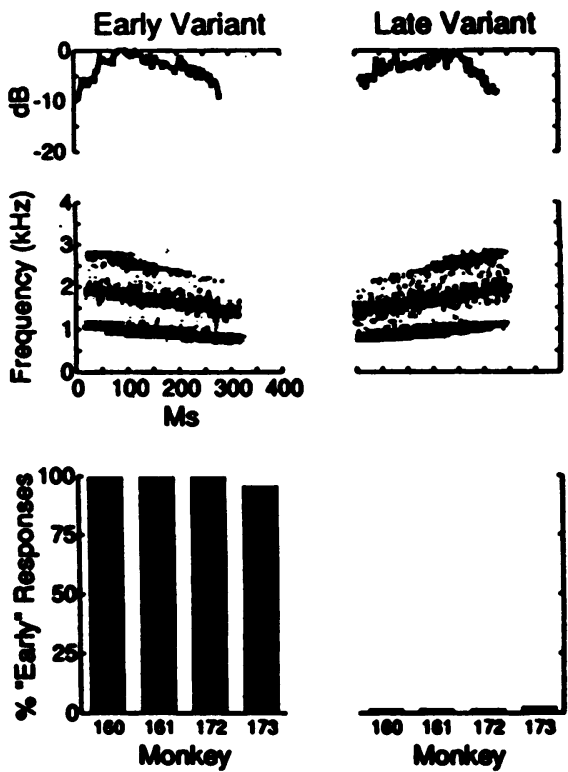

Figure 8. Sonogram for synthetic early (upper left) and late (upper right) inflected coo calls. The contours at the top of the panel represent the amplitude changes while those just below represent the frequency changes for the fundamental and harmonics. The inflection has been removed from the frequency change. Responses to the calls in lower panels as described for Figure 4. Animals responded to the early variant as an early inflected call, but not similarly to the late variant. From "The Significant Features of Japanese Macaque Coo Sounds: A Psychological Study" by B. J. May, D. B. Moody, and W. C. Stebbins, 1988, Animal Behavior, 36, pp. 1432-1444. Copyright 1988 by Bailliere Tindall. Reprinted by permission.

and Nat Schoenfeld with a most important contribution from the book Principles of Psychology. In the substantial legacy that we have from both Fred Keller and Fred Skinner in the application of these principles to education, we may sometimes overlook the vast implications of the experimental analysis of behavior for related basic biological science disciplines. We have drawn examples from only two-neurophysiology and evolutionary biology. There are others that have profited from this rigorous and systematic approach to the study of behavior.

\section{REFERENCES}

Beecher, M. D., Peterson, M. R., Zoloth, S. R., Moody, D. B., \& Stebbins, W. C. (1979). Macaque perception of conspecific communication sounds. Brain, Behavior, and Evolution, 16, 443460.

Green, S. (1975). The variation of vocal pattern with social situation in the Japanese monkey (Macaca fuscata): A field study. In L. Rosenblum (Ed.), Primate behavior (Vol. 4, pp. 1-102). New York: Academic Press.

Heffner, H. E., \& Heffner, R. S. (1984). Temporal lobe lesions and perception of species-specific vocalizations by macaques. Science, $226,75-76$.

Herrnstein, R. J., \& Van Sommers, P. (1962). Method for sensory scaling with animals. Science, 135, 40-41.

Keller, F. S., \& Schoenfeld, W. N. (1950). Principles of psychology. New York: Appleton-Century-Crofts.

Kuhl, P. K. (1986). Theoretical contributions of tests on animals to the special-mechanisms debate in speech. Experimental Biology, 45, 233265.

Liberman, A. M. (1982). On finding that speech is special. American Psychologist, 37, 148-167.

May, B. J., Moody, D. B., \& Stebbins, W. C. (1988). The significant features of Japanese macaque coo sounds: A psychological study. Animal Behavior, 36, 1432-1444.

May, B. J., Moody, D. B., \& Stebbins, W. C. (1989). Categorical perception of conspecific communication sounds by Japanese macaques (Macaca fuscata). Journal of the Acoustical Society of America, 85, 837-847.

Peterson, M. R., Beecher, M. D., Zoloth, S. R., Moody, D. B., \& Stebbins, W. C. (1978). Neural lateralization of species-specific vocalizations by Japanese macaques (Macaca fuscata). Science, 202, 324-327.

Skinner, B. F. (1938). Behavior of organisms. New York: Appleton-Century-Crofts.

Smith, D. W., Moody, D. B., Stebbins, W. C., \& Norat, M. A. (1987). Effects of outer hair cell loss on the frequency selectivity of the patas monkey auditory system. Hearing Research, 29, 125138.

Stebbins, W. C. (in press). Perception in animal behavior. In M. A. Berkley \& W. C. Stebbins (Eds.), Comparative perception (Vol. 1). New York: Wiley.

Stebbins, W. C., \& Berkley, M. A. (Eds.). (in press). Comparative Perception (Vol. 2). New York: Wiley.

Stebbins, W. C., \& Moody, D. B. (1979). Comparative behavioral toxicology. Neurobehavioral Toxicology and Teratology, 1 (Suppl. 1), 33-44. 